\title{
OVEREXPRESSION OF APOBEC3B ENHANCES INVASIVENESS OF HEPATOCELLULAR CARCINOMA
}

This study aims at revealing the roles of APOBEC3B (A3B) in human hepatocellular carcinoma (HCC). HCC is a leading cancer worldwide and specifically prevalent in Southeast Asia, due to prevalent hepatitis B virus (HBV) infection. Recent evidence has implicated A3B, a DNA deaminase, as a source of mutations in breast, cervical and bladder cancers. For example, A3B was found upregulated and active in the majority of breast cancer cell lines, and its upregulation in tumors correlated with increased C-to-T transition and overall base substitution mutations. $\mathrm{A} 3 \mathrm{~B}$ is one of seven APOBEC3 deaminases, which is responsible for providing innate immunity to a large number of DNA-based viruses. All these enzymes exhibit DNA deaminase activity. Transgenic expression of APOBEC1 and AID can induce tumor formation in mice which suggests links between mutagenesis, immunity and viral infection in cancer development. However, their roles in HCC remains largely undiscovered.

We sequenced 16 pairs of $\mathrm{HCC}$ and corresponding non-tumorous livers using whole transcriptome sequencing (RNA-seq) and exome sequencing. We found that A3B expression was significantly overexpressed in $\mathrm{HCC}$ as compared to the corresponding non-tumorous liver for 13 of the 16 samples $(81.3 \%$ ) ( $\mathrm{p}<0.001)$. Moreover, the frequency of C-to-U and G-to-A mutations was associated with the metastasis status and significant association was detected at RNA level ( $\mathrm{p}$ $=0.015)$ while a similar trend was also observed at the DNA level $(\mathrm{p}=0.059$ for $\mathrm{C}>\mathrm{T}$ plus $\mathrm{G}>\mathrm{A}$ events).

To validate the expression in a larger cohort of human HCCs, we performed real-time quantitative PCR (qPCR) on 55 pairs of human HCCs. We consistently observed a frequent (70.9\%) and significant overexpression $(\mathrm{p}<0.001)$ of A3B in HCCs. The result was also confirmed at the protein level by immunohistochemistry and western blotting. Clinicalpathological analysis revealed that A3B overexpression in HCCs was significantly associated with more aggressive tumor behavior including the presence of tumor microsatellite formation $(\mathrm{p}=0.009)$, venous invasion $(\mathrm{p}=0.021)$, direct invasion into the adjacent liver tissue $(\mathrm{p}=0.033)$ and more advanced tumor stages ( $\mathrm{p}=0.017)$. In addition, overexpression of A3B in HCCs positively correlated with the presence of $\mathrm{HBX}$ truncation $(\mathrm{P}=0.017)$. We also examined the $\mathrm{A} 3 \mathrm{~B}$ expression in a panel of HCC cell lines and found that the A3B mRNA levels were higher in the HCC cell lines with higher metastatic potential. In vitro cell migration and invasion assays also showed that the HCC cells which had higher expression levels of A3B had higher migratory and invasive abilities. In conclusion, we demonstrated that $\mathrm{A} 3 \mathrm{~B}$ was overexpressed in $\mathrm{HCC}$ and its overexpression was associated with more aggressive tumor behavior. A3B overexpression might enhance the metastatic potential of HCC cells. 